\title{
BOUNDS FOR THE RAYLEIGH QUOTIENT AND THE SPECTRUM OF SELF-ADJOINT OPERATORS *
}

\author{
PEIZHEN ZHU ${ }^{\dagger}$ MERICO E. ARGENTATI ${ }^{\dagger \ddagger}$ AND ANDREW V. KNYAZEV ${ }^{\dagger} \S$
}

\begin{abstract}
The absolute change in the Rayleigh quotient (RQ) is bounded in this paper in terms of the norm of the residual and the change in the vector. If $x$ is an eigenvector of a self-adjoint bounded operator $A$ in a Hilbert space, then the RQ of the vector $x$, denoted by $\rho(x)$, is an exact eigenvalue of $A$. In this case, the absolute change of the RQ $|\rho(x)-\rho(y)|$ becomes the absolute error for an eigenvalue $\rho(x)$ of $A$ approximated by the RQ $\rho(y)$ on a given vector $y$. There are three traditional kinds of bounds for eigenvalue errors: a priori bounds via the angle between vectors $x$ and $y$; a posteriori bounds via the norm of the residual $A y-\rho(y) y$ of vector $y$; mixed type bounds using both the angle and the norm of the residual. We propose a unifying approach to prove known bounds of the spectrum, analyze their sharpness, and derive new sharper bounds. The proof approach is based on novel RQ vector perturbation identities.
\end{abstract}

Key words. angles, perturbation, error analysis, Rayleigh quotient, eigenvalue.

AMS subject classification. 15A42, 15A60, 65F 35.

1. Introduction. Let $A$ be a bounded self-adjoint operator in a real or complex Hilbert space $\mathcal{H}$. For a nonzero vector $x$, the Rayleigh quotient (RQ) is defined by

$$
\rho(x)=\rho(x, A)=\frac{\langle x, A x\rangle}{\langle x, x\rangle}
$$

and the corresponding residual vector is denoted by $r(x)=r(x, A)=A x-\rho(x) x$, where $\langle\cdot, \cdot\rangle$ is an inner product, associated with a norm $\|\cdot\|^{2}=\langle\cdot, \cdot\rangle$. The acute angle between two nonzero vectors $x$ and $y$ is denoted by

$$
\angle\{x, y\}=\arccos \frac{|\langle x, y\rangle|}{\|x\|\|y\|} .
$$

We are interested in RQ vector perturbation bounds. Specifically, for nonzero vectors $x$ and $y$, we want to bound the following quantity, $|\rho(x)-\rho(y)|$, in terms of $\angle\{x, y\},\|r(x)\|$, and $\|r(y)\|$. If $x$ is an eigenvector of $A$, then the RQ of $x$ is an exact eigenvalue of $A$ and $r(x)=0$. In this case, the absolute change of the RQ $|\rho(x)-\rho(y)|$ becomes the absolute error in the eigenvalue $\rho(x)$ of $A$. The RQ is often used to approximate points of the spectrum $\Sigma(A)$ of $A$.

Known bounds of the spectrum are traditionally classified depending on the terms that appear in the bounds. Let $\lambda \in \Sigma(A)$ be approximated by $\rho(y)$, i.e., the absolute approximation error is $|\lambda-\rho(y)|$. Bounds of $|\lambda-\rho(y)|$ that are based on the norm of the residual $\|r(y)\|$ are called "a posteriori" bounds, since the residual $r(y)$ and its norm can typically be computed for the given vector $y$.

If $\lambda$ is an eigenvalue with the corresponding eigenvector $x$, bounds for $|\lambda-\rho(y)|$ that rely on the angle $\angle\{x, y\}$ are called "a priori," since the eigenvector $x$ is usually

*October 29, 2018. Preliminary posted at http://arxiv.org/abs/math/0610498. This material is based upon work partially supported by the National Science Foundation under Grant No. 1115734.

${ }^{\dagger}$ Department of Mathematical and Statistical Sciences; University of Colorado Denver, P.O. Box 173364, Campus Box 170, Denver, CO 80217-3364, USA.

$\ddagger$ (peizhen.zhu,merico.argentati,andrew.knyazev)[at]ucdenver.edu

$\S$ Mitsubishi Electric Research Laboratories; 201 Broadway Cambridge, MA 02139

Thttp://www.merl.com/people/?user=knyazev and http://math.ucdenver.edu/ aknyazev/ 
not explicitly known, and some a priori information about $x$ needs to be used to bound the angle $\angle\{x, y\}$. In the context of Finite Element Method error bounds, where $x$ and $y$ are functions, such information is usually associated with the smoothness of the function $x$; see, e.g., [8] and references there. Finally, we call a bound "a mixed type" if it involves both terms, the residual norm $\|r(y)\|$ and the angle $\angle\{x, y\}$.

Sharp bounds of the spectrum approximation error are very important in the theory of numerical solution of self-adjoint eigenvalue problems. Computable a posteriori bounds allow one to obtain approximations from below and above to points of the spectrum. A priori and mixed type bounds give one an opportunity to determine the quality of approximation of a point of the spectrum by using the RQ.

We revisit this classical topic of research for three reasons. Our first goal is to carefully examine a posteriori and a priori bounds together, to understand what they have in common and why they are so different. Second, we are interested in discovering a general framework for deriving bounds of $|\rho(x)-\rho(y)|$ for arbitrary vectors $x$ and $y$ where known bounds of the spectrum approximation error become corollaries. Last, but not least, bounds of $|\rho(x)-\rho(y)|$ for arbitrary vectors $x$ and $y$ are practically important on their own, e.g., as a tool for deriving sharp convergence rate bounds of eigenvalue solvers and analyzing the effects of inexact computations. We anticipate that our results will lead to new proof techniques and sharp bounds of accuracy of Ritz and harmonic (e.g., [13]) Ritz pairs, which are important in applications.

We start, in section 2, by reviewing three known bounds of the spectrum approximation error, each representing a priori, a posteriori, and mixed types, correspondingly. In section 3, we derive new identities and bounds for the change in the $\mathrm{RQ}$ with respect to the change of the vectors, using an orthogonal projector on the subspace $\operatorname{span}\{x, y\}$. The idea of such a "low-dimensional" analysis has been found fruitful before, e.g., in [4]. We observe that in the case $\operatorname{dim} \mathcal{H}=2$ all three bounds, which we want to reinvent, turn into the same identity. In section 4 , we derive the a priori and mixed type bounds using this identity, using a single inequality, different for each bound, only at the last step. This unifying proof technique allows us to easily specify the circumstances where the bounds are sharp. We dedicate section 5 to a posteriori bounds. Our "low-dimensional" analysis not only allows us to find a novel proof of a well known a posteriori bound, but also gives us an opportunity to improve it, and obtain several new sharper results. Our bonus section 6 touches the topic of improving known $\sin (2 \theta)$ and $\tan (\theta)$ error bounds for eigenvectors.

2. Short review of some known error bounds for eigenvalues. Let $y$ be an approximation to an eigenvector $x$ of the operator $A$, corresponding to the eigenvalue $\rho(x)$ of $A$. An a priori bound involves a constant and the square of the sine of the angle between the eigenvector $x$ and the vector $y$, see, e.g., $[6,7,11]$,

$$
|\lambda-\rho(y)| \leq\left(\Sigma_{\max }(A)-\Sigma_{\min }(A)\right) \sin ^{2}(\angle\{x, y\}),
$$

where we denote $\lambda=\rho(x)$, and $\Sigma_{\max }(A)$ and $\Sigma_{\min }(A)$ denote the largest and the smallest points of the spectrum $\Sigma(A)$ of $A$, correspondingly.

The mixed type bound shown in $[5,14]$ is in terms of the norm of the residual vector and the tangent of the angle between vectors $x$ and $y$, i.e.,

$$
|\lambda-\rho(y)| \leq \frac{\|r(y)\|}{\|y\|} \tan (\angle\{x, y\}) .
$$

There is no constraint on the location of the eigenvalue $\rho(x)=\lambda$ relative to $\rho(y)$ in (2.1) and (2.2). Bounds (2.1) and (2.2) hold for any nonzero vector $y$ and 
any eigenvector $x$. The next bound we review removes the eigenvector $x$ from the picture, but instead requires having some information about the spectrum of $A$ in the neighborhood of $\rho(y)$. This a posteriori bound involves the square of the norm of the residual vector and the gap between $\rho(y)$ and the spectrum $\Sigma(A)$ of the operator $A$.

Let real numbers $\alpha<\beta$ be such that $\Sigma(A) \cap(\alpha, \beta)=\emptyset$. Using the spectral decomposition of the self-adjoint bounded operator $A$, and the fact that the quadratic polynomial $(t-\alpha)(t-\beta) \geq 0$ for any real $t$ outside of the interval $(\alpha, \beta)$, it is easy to see that $(A-\alpha I)(A-\beta I) \geq 0$, where $I$ is the identity, i.e., that $\langle A y-\alpha y, A y-\beta y\rangle \geq 0$ for any vector $y$. Elementary algebraic manipulations for nonzero $y$ show that this inequality is equivalent to the famous bound attributed to Temple, see $[3,16]$,

$$
(\beta-\rho(y))(\rho(y)-\alpha) \leq \frac{\|r(y)\|^{2}}{\|y\|^{2}} .
$$

In the nontrivial case $\alpha<\rho(y)<\beta$, we choose the scalars $\alpha \in \Sigma(A)$ and $\beta \in \Sigma(A)$ as the nearest points of the spectrum below and above $\rho(y)$. This results in the tightest bound (2.3), since its left-hand side is monotonic in $\alpha$ and $\beta$. In other words, for given $A$ and $y$ with $\rho(y) \notin \Sigma(A)$, there exist $\alpha \in \Sigma(A)$ and $\beta \in \Sigma(A)$ such that $\alpha<\rho(y)<\beta$ and (2.3) holds. Bound (2.3) is sharp if $\alpha$ and $\beta$ are eigenvalues of $A$ and the vector $y$ is a linear combination of the corresponding eigenvectors.

Next, for $A$ and $y$ with $\rho(y) \notin \Sigma(A)$, there exist $\lambda \in \Sigma(A)$ and real scalars $a$ and $b$ such that $a<\rho(y)<b$ and $a \leq \lambda \leq b$, while $\Sigma(A) \cap(a, \lambda)=\emptyset$ and $\Sigma(A) \cap(\lambda, b)=\emptyset$, and Kato-Temple's inequality (see, e.g., [3] and [10, Theorem VIII.5, p. 84]) holds,

$$
-\frac{1}{\rho(y)-a} \frac{\|r(y)\|^{2}}{\|y\|^{2}} \leq \rho(y)-\lambda \leq \frac{1}{b-\rho(y)} \frac{\|r(y)\|^{2}}{\|y\|^{2}} .
$$

Indeed, in the case where $\lambda$ is located to the right of $\rho(y)$ we take $\alpha=a$ and $\beta=\lambda$ in (2.3) to get the lower bound of (2.4). In the opposite case, $\lambda<\rho(y)$, we take $\alpha=\lambda$ and $\beta=b$ to obtain the upper bound of (2.4). If $\lambda \in(a, b)$, then $\lambda=\Sigma(A) \cap(a, b)$, and thus $\lambda$ is unique and in fact is an isolated eigenvalue.

Let $a \in \Sigma(A)$ and $b \in \Sigma(A)$ in (2.4) and denote $\delta=\min _{\eta \in\{\Sigma(A) \backslash\{\lambda\}\}}|\eta-\rho(y)|$. Checking different scenarios $\lambda=a, \lambda=b$, and $\lambda \in(a, b)$ in (2.4), we observe that inequalities (2.4) imply, see, e.g., $[1,12,15]$, the existence of $\lambda \in \Sigma(A)$ such that

$$
|\lambda-\rho(y)| \leq \frac{1}{\delta} \frac{\|r(y)\|^{2}}{\|y\|^{2}} .
$$

In bound (2.5), $\lambda \in \Sigma(A)$ may be not unique and in general does not have to be an isolated point of the spectrum, or even an eigenvalue. Bound (2.5) is sharp if at least one of the scalars $\rho(y)-\delta$ or $\rho(y)+\delta$ is an eigenvalue of $A$ and the vector $y$ is a linear combination of the corresponding eigenvector and an eigenvector corresponding to $\lambda$.

The Krylov-Weinstein [17, p. 321] (Bauer-Fike [12, Theorem 3.6]) theorem states the existence (but not necessarily uniqueness) of $\lambda \in \Sigma(A)$ such that

$$
|\lambda-\rho(y)| \leq \frac{\|r(y)\|}{\|y\|} .
$$

It follows directly from (2.3), by choosing $\lambda=\alpha \in \Sigma(A)$ or $\lambda=\beta \in \Sigma(A)$, whichever is closer to $\rho(y)$. As in (2.5), $\lambda \in \Sigma(A)$ does not have to be an isolated point of the spectrum, or even an eigenvalue. Bound (2.6) turns into equality if bound (2.3) is equality and, in addition, $\rho(y)-\alpha=\beta-\rho(y)$ in (2.3). 
This derivation of (2.6) from bound (2.3) following [1, Corollary 6.20, p. 303] is not so well known. We conclude that bound (2.3) is the most fundamental a posteriori bound, since all other a posteriori bounds, reviewed here, can be derived from it.

The main goal of the rest of the paper is to revisit bounds (2.1), (2.2), and (2.3). We propose a new unifying approach to prove them, analyze their sharpness, and derive some new sharper bounds. The proof approach is based on novel RQ vector perturbation identities presented in the next section.

3. Key identities for the RQ. We start with a few trivial but key properties of the RQ. Then we derive a couple of simple expressions for the norm of the residual. Finally, our main results, several identities for the absolute change in the RQ, follow.

Let $A$ be a bounded self-adjoint operator on a real or complex Hilbert space $\mathcal{H}$. Let $S$ denote a subspace of $\mathcal{H}$ and $P_{S}$ be an orthogonal projector on $S$. Let $A_{S}=\left.\left(P_{S} A\right)\right|_{S}$ denote the restriction of the operator $P_{S} A$ to the subspace $S$. For a nonzero vector $x \in S$, we denote $\rho\left(x, A_{S}\right)=\left\langle x, A_{S} x\right\rangle /\langle x, x\rangle$ and $r\left(x, A_{S}\right)=A_{S} x-\rho\left(x, A_{S}\right) x \in S$. We start with a couple of trivial, but extremely important, for our approach, lemmas.

Lemma 3.1. If $x \in S$ then $\rho\left(x, A_{S}\right)=\rho(x)$.

Proof. The orthogonal projector $P_{S}$ is self-adjoint, so

$$
\rho\left(x, A_{S}\right)=\frac{\left\langle x, P_{S} A x\right\rangle}{\langle x, x\rangle}=\frac{\langle x, A x\rangle}{\langle x, x\rangle}=\rho(x) .
$$

Lemma 3.2. If $x \in S$ then $r\left(x, A_{S}\right)=P_{S} r(x)$.

Proof. Directly by the definition and Lemma 3.1, we obtain

$$
r\left(x, A_{S}\right)=A_{S} x-\rho\left(x, A_{S}\right) x=P_{S} A x-\rho(x) x=P_{S}(A x-\rho(x) x)=P_{S} r(x) .
$$

口

Corollary 3.3. If $x \in S$ is an eigenvector of $A$, it is also an eigenvector of $A_{S}$, corresponding to the same eigenvalue $\rho(x)=\rho\left(x, A_{S}\right)$.

Proof. By Lemma 3.1, $\rho(x)=\rho\left(x, A_{S}\right)$. For an eigenvector $x$ of $A$, the corresponding eigenvalue is $\rho(x)=\rho\left(x, A_{S}\right)$, so $r(x)=0$, and $r\left(x, A_{S}\right)=P_{S} r(x)=0$, by Lemma 3.2. Thus $x$ is also an eigenvector of $A_{S}$, with the same eigenvalue.

In the rest of the paper we always assume or prove that $\operatorname{dim} S=2$.

Now, we collect some basic identities, using the eigenvectors of $A_{S}$. Let us denote ${ }^{1}$ $\mu=\lambda_{\max }\left(A_{S}\right)=\rho\left(u_{1}\right)$ and $\nu=\lambda_{\min }\left(A_{S}\right)=\rho\left(u_{2}\right)$, where $u_{1}$ and $u_{2}$ are orthogonal eigenvectors of the operator $A_{S}$. Let $P_{i}$ with $i=1,2$ denote the orthogonal projector on the subspace $\operatorname{span}\left\{u_{i}\right\}$. Assuming a nontrivial case $P_{i} x \neq 0$ for $i=1,2$, we evidently have $\angle\left\{x, u_{i}\right\}=\angle\left\{x, P_{i} x\right\}$. Now, since $x \in S$ and $\operatorname{dim} S=2$,

$$
\rho(x)=\frac{\left\langle x, A_{S} x\right\rangle}{\langle x, x\rangle}=\frac{\left\langle P_{1} x, A_{S} P_{1} x\right\rangle+\left\langle P_{2} x, A_{S} P_{2} x\right\rangle}{\left\langle P_{1} x, P_{1} x\right\rangle+\left\langle P_{2} x, P_{2} x\right\rangle}=\frac{\mu\left\langle P_{1} x, P_{1} x\right\rangle+\nu\left\langle P_{2} x, P_{2} x\right\rangle}{\left\langle P_{1} x, P_{1} x\right\rangle+\left\langle P_{2} x, P_{2} x\right\rangle}
$$

where $x=P_{1} x+P_{2} x$ and $P_{1} P_{2}=P_{1} A_{S} P_{2}=0$. Therefore,

$$
\mu-\rho(x)=(\mu-\nu) \sin ^{2}\left(\angle\left\{x, u_{1}\right\}\right) \text { and } \rho(x)-\nu=(\mu-\nu) \sin ^{2}\left(\angle\left\{x, u_{2}\right\}\right) .
$$

Evidently, in a two-dimensional space,

$$
\cos \left(\angle\left\{x, u_{1}\right\}\right)=\sin \left(\angle\left\{x, u_{2}\right\}\right) \text { and } \cos \left(\angle\left\{x, u_{2}\right\}\right)=\sin \left(\angle\left\{x, u_{1}\right\}\right) .
$$

\footnotetext{
${ }^{1}$ We intentionally introduce new notation $\lambda_{\max }\left(A_{S}\right)=\Sigma_{\max }\left(A_{S}\right)$ and $\lambda_{\min }\left(A_{S}\right)=\Sigma_{\min }\left(A_{S}\right)$ to underline the fact that these points of the spectrum $\Sigma\left(A_{S}\right)$ are actually eigenvalues.
} 
3.1. Identities for the norm of the residual $r\left(x, A_{S}\right)=P_{S} r(x)$. Our main identity is in the following lemma.

Lemma 3.4. Let $x \in S$ and $\operatorname{dim} S=2$. Then

$$
\left[\lambda_{\max }\left(A_{S}\right)-\rho(x)\right]\left[\rho(x)-\lambda_{\min }\left(A_{S}\right)\right]=\frac{\left\|P_{S} r(x)\right\|^{2}}{\|x\|^{2}} .
$$

Proof. Let us denote $\mu=\lambda_{\max }\left(A_{S}\right)$ and $\nu=\lambda_{\min }\left(A_{S}\right)$. By Lemmas 3.1 and 3.2, identity (3.3) can be equivalently rewritten as

$$
\left[\mu-\rho\left(x, A_{S}\right)\right]\left[\rho\left(x, A_{S}\right)-\nu\right]=\frac{\left\|r\left(x, A_{S}\right)\right\|^{2}}{\|x\|^{2}} .
$$

Since $\left\langle r\left(x, A_{S}\right), x\right\rangle=0$, we have $\left\|r\left(x, A_{S}\right)\right\|^{2}=\left\|A_{S} x\right\|^{2}-\rho^{2}\left(x, A_{S}\right)\|x\|^{2}$ and thus

$$
\begin{aligned}
\frac{\left\|r\left(x, A_{S}\right)\right\|^{2}}{\|x\|^{2}}-\left(\mu-\rho\left(x, A_{S}\right)\right)\left(\rho\left(x, A_{S}\right)-\nu\right) & =\frac{\left\|A_{S} x\right\|^{2}}{\|x\|^{2}}-(\mu+\nu) \rho\left(x, A_{S}\right)+\mu \nu \\
& =\frac{\left\langle A_{S} x-\mu x, A_{S} x-\nu x\right\rangle}{\|x\|^{2}} \\
& =0
\end{aligned}
$$

where $\left(A_{S}-\mu\right)\left(A_{S}-\nu\right)=0$ is the minimal polynomial of $A_{S}$, since $\operatorname{dim} S=2$ and $\mu$ and $\nu$ are the eigenvalues of $A_{S}$. $\mathrm{v}$

We also mention the following identity, relying on eigenvectors $u_{i}$ of $A_{S}$.

Lemma 3.5. Let $x \in S$ and $\operatorname{dim} S=2$. Then

$$
\frac{1}{2}\left[\lambda_{\max }\left(A_{S}\right)-\lambda_{\min }\left(A_{S}\right)\right] \sin \left(2 \angle\left\{x, u_{i}\right\}\right)=\frac{\left\|P_{S} r(x)\right\|}{\|x\|}, i=1,2 .
$$

Proof. Applying (3.1) in (3.3), and taking the square root, we get

$$
(\mu-\nu) \sin \left(\angle\left\{x, u_{1}\right\}\right) \sin \left(\angle\left\{x, u_{2}\right\}\right)=\frac{\left\|P_{S} r(x)\right\|}{\|x\|} .
$$

By $(3.2), \sin \left(\angle\left\{x, u_{2}\right\}\right)=\cos \left(\angle\left\{x, u_{1}\right\}\right)$, so the statement of the lemma for $i=1$ follows from the trigonometry identity for the sine of a double angle. Identities (3.2) also imply $\sin \left(2 \angle\left\{x, u_{1}\right\}\right)=\sin \left(2 \angle\left\{x, u_{2}\right\}\right)$, even though it looks counterintuitive. $\mathrm{D}$

3.2. Identities for the absolute change in the RQ. Here we prove our main tangent- and sine-based identities.

In the remainder of the paper, for given linearly independent vectors $x$ and $y$ in $\mathcal{H}$ we always define the subspace $S$ as $S=\operatorname{span}\{x, y\}$.

REMARK 3.6. Let $0<\angle\{x, y\}<\pi / 2$. We note the following useful identities

$\frac{\|r(x)\|}{\|x\|} \frac{\cos (\angle\{r(x), y\})}{\cos (\angle\{x, y\})}=\frac{|\langle r(x), y\rangle|}{|\langle x, y\rangle|}=\frac{\left|\left\langle P_{S} r(x), y\right\rangle\right|}{|\langle x, y\rangle|}=\frac{\left\|P_{S} r(x)\right\|}{\|x\|} \frac{\cos \left(\angle\left\{P_{S} r(x), y\right\}\right)}{\cos (\angle\{x, y\})}$

and

$$
\frac{\left\|P_{S} r(x)\right\|}{\|x\|} \frac{\cos \left(\angle\left\{P_{S} r(x), y\right\}\right)}{\cos (\angle\{x, y\})}=\frac{\left\|P_{S} r(x)\right\|}{\|x\|} \tan (\angle\{x, y\}),
$$


where $\cos \left(\angle\left\{P_{S} r(x), y\right\}\right)=\sin (\angle\{x, y\})$. Indeed, $0=\langle r(x), x\rangle=\left\langle P_{S} r(x), x\right\rangle$, i.e., vectors $P_{S} r(x) \in S$ and $x \in S$ are orthogonal in $S$, and also $y \in S$, where $\operatorname{dim} S=2$. Denoting the orthogonal projector on the subspace $\operatorname{span}\{y\}$ by $P_{y}$ and using $\angle\left\{P_{y} r(x), y\right\}=0$, we also get

$$
\frac{|\langle r(x), y\rangle|}{|\langle x, y\rangle|}=\frac{\left|\left\langle P_{y} r(x), y\right\rangle\right|}{|\langle x, y\rangle|}=\frac{\left\|P_{y} r(x)\right\|}{\|x\|} \frac{\cos \left(\angle\left\{P_{y} r(x), y\right\}\right)}{\cos (\angle\{x, y\})}=\frac{\left\|P_{y} r(x)\right\|}{\|x\| \cos (\angle\{x, y\})} .
$$

Theorem 3.7. Let $0<\angle\{x, y\}<\pi / 2$ and let us denote

$$
\Xi_{ \pm}=\left|\frac{\left\|P_{S} r(x)\right\|}{\|x\|} \pm \frac{\left\|P_{S} r(y)\right\|}{\|y\|}\right| \tan (\angle\{x, y\}) .
$$

We always have

$$
\Xi_{-} \leq|\rho(x)-\rho(y)| \leq \Xi_{+} .
$$

Now, let us denote $a=\langle x, r(y)\rangle, b=\langle r(x), y\rangle$, and $c=\langle x, y\rangle \neq 0$ and assume that $a / c$ or $b / c$ is real. Then $|\rho(x)-\rho(y)|=\Xi_{-}$if $b \neq 0$ and $a / b \geq 0$ (or $a \neq 0$ and $b / a \geq 0)$; otherwise, $|\rho(x)-\rho(y)|=\Xi_{+}$.

Proof. We consider only the nontrivial case where at least one of the vectors $x$ and $y$ is not an eigenvector of $A_{S}$. Let it be, e.g., $y$, so that the set $y$ and $P_{S} r(y) \neq 0$ form an orthogonal basis of the subspace $S$. Then $a=\langle x, r(y)\rangle=\left\langle x, P_{S} r(y)\right\rangle \neq 0$, since $\angle\{x, y\} \neq 0$. By elementary calculations, we obtain

$$
\begin{aligned}
(\rho(x)-\rho(y))\langle x, y\rangle & =\langle\rho(x) x, y\rangle-\langle x, \rho(y) y\rangle+\langle x, A y\rangle-\langle A x, y\rangle \\
& =\langle x, r(y)\rangle-\langle r(x), y\rangle,
\end{aligned}
$$

thus $\rho(x)-\rho(y)=(a-b) / c$ and $|\rho(x)-\rho(y)|=|a-b| /|c|$. In a complex space, the RQ remains real, since $A$ is Hermitian, but the scalar products $a, b$, and $c$ may be not real, so in general we have, by the triangle inequality for complex scalars, that ||$a|-| b|| \leq|a-b| \leq|a|+|b|$, where the inequalities are strict unless $b / a$ is a real number. On the other hand, $\rho(x)-\rho(y)=(a-b) / c$ is always real, so $b / a$ is real iff $a / c$ or $b / c$ is real, which is the second assumption of the theorem. Under this assumption, ||$a|-| b||=|a-b|$, if $b / a \geq 0$, or $|a-b|=|a|+|b|$, if $b / a \leq 0$, which completely characterizes the cases where inequalities in (3.5) turn into equalities.

The statements of the theorem now follow directly from Remark 3.6.

Lemma 3.8. Let $\angle\{x, y\}>0$. For both $i=1$ and 2 , we have

$$
|\rho(x)-\rho(y)|=[\mu-\nu] \sin \left(\angle\left\{x, u_{i}\right\}+\angle\left\{y, u_{i}\right\}\right)\left|\sin \left(\angle\left\{x, u_{i}\right\}-\angle\left\{y, u_{i}\right\}\right)\right| .
$$

Proof. Identities (3.1) and (3.2) imply

$$
|\rho(x)-\rho(y)|=[\mu-\nu]\left|\cos ^{2}\left(\angle\left\{x, u_{i}\right\}\right)-\cos ^{2}\left(\angle\left\{y, u_{i}\right\}\right)\right|,
$$

which leads directly to identity (3.6) using elementary trigonometry. $\square$

Theorem 3.9. Let $\angle\{x, y\}>0$. Let us for $i=1$ or 2 denote

$$
\Psi_{ \pm}=\left[\lambda_{\max }\left(A_{S}\right)-\lambda_{\min }\left(A_{S}\right)\right]\left|\sin \left(\angle\left\{x, u_{i}\right\} \pm \angle\left\{y, u_{i}\right\}\right)\right| \sin (\angle\{x, y\}),
$$

and let $C=\left\langle x, u_{1}\right\rangle\left\langle u_{2}, x\right\rangle\left\langle u_{1}, y\right\rangle\left\langle y, u_{2}\right\rangle$. We always have $\Psi_{-} \leq|\rho(x)-\rho(y)| \leq \Psi_{+}$. If the scalar $C$ is not real then both inequalities on the left and right are strict. Moreover, if $C \geq 0(C \leq 0)$ then we have equality for the lower (upper) bound. 
Proof. Identity (3.6) makes the statement of the theorem equivalent to

$$
\left|\sin \left(\angle\left\{x, u_{i}\right\}-\angle\left\{y, u_{i}\right\}\right)\right| \leq \sin \angle\{x, y\} \leq \sin \left(\angle\left\{x, u_{i}\right\}+\angle\left\{y, u_{i}\right\}\right) .
$$

For simplicity of notation, let us assume, without loss of generality, that all vectors involved are normalized, i.e., $\left\|u_{1}\right\|=\left\|u_{2}\right\|=\|x\|=\|y\|=1$. Using the representations of the vectors $x$ and $y$ with respect to the orthonormal basis $u_{1}$ and $u_{2}$ for the subspace $S$, we obtain by direct computation

$$
\sin ^{2} \angle\{x, y\}=\cos ^{2} \angle\left\{x, u_{1}\right\} \cos ^{2} \angle\left\{y, u_{2}\right\}+\cos ^{2} \angle\left\{x, u_{2}\right\} \cos ^{2} \angle\left\{y, u_{1}\right\}-2 \Re(C)
$$

and

$$
|C|=\cos \angle\left\{x, u_{1}\right\} \cos \angle\left\{x, u_{2}\right\} \cos \angle\left\{y, u_{1}\right\} \cos \angle\left\{y, u_{2}\right\} .
$$

Evidently, $-|C| \leq \Re(C) \leq|C|$, and if $C \geq 0(C \leq 0)$ then $\Re(C)=|C|(\Re(C)=-|C|)$, which proves bounds (3.7) and also gives conditions for their sharpness. $\square$

REMARK 3.10. An alternative proof of Theorem 3.9 is based on the fact that angles between subspaces describe a metric, see, e.g. [18] and references there, so

$$
|\angle\{x, u\}-\angle\{y, u\}| \leq \angle\{x, y\} \leq|\angle\{x, u\}+\angle\{y, u\}| .
$$

Theorem 3.9 trivially implies the bound

$$
|\rho(x)-\rho(y)| \leq\left[\lambda_{\max }\left(A_{S}\right)-\lambda_{\min }\left(A_{S}\right)\right] \sin (\angle\{x, y\})
$$

that can be found for the real case in [7].

Finally, we apply our results above to an important special case.

Corollary 3.11. Let $x$ be an eigenvector of $A$ and $\rho(x)=\lambda$. Then

$$
|\lambda-\rho(y)|=\left[\lambda_{\max }\left(A_{S}\right)-\lambda_{\min }\left(A_{S}\right)\right] \sin ^{2}(\angle\{x, y\}) .
$$

If, in addition $\angle\{x, y\}<\pi / 2$, then

$$
|\lambda-\rho(y)|=\frac{\left\|P_{S} r(y)\right\|}{\|y\|} \tan (\angle\{x, y\}) .
$$

If also $\eta \neq \lambda$ denotes an eigenvalue of $A_{S}$, then

$$
\tan (\angle\{x, y\})=\frac{1}{|\eta-\rho(y)|} \frac{\left\|P_{S} r(y)\right\|}{\|y\|} .
$$

Proof. Since $x$ is an eigenvector of $A$ it is also an eigenvector of $A_{S}$, with the same eigenvalue $\rho(x)=\lambda$, by Corollary 3.3. The statements (3.9) and (3.10) follow directly from Theorems 3.7 and 3.9 , correspondingly. Identity (3.11) is derived from (3.3) and (3.10). Finally, the assumption $\angle\{x, y\}>0$ is dropped, since in the case $\angle\{x, y\}=0$ all the statements trivially hold, where both sides vanish.

The next section is entirely based on Corollary 3.11. In the rest of the paper, we do not use our identities and bounds for $|\rho(x)-\rho(y)|$ for arbitrary nonzero vectors $x$ and $y$. We want to highlight again, that these results have merit on their own, not just as a tool for derivation of eigenvalue error bounds. 
4. Deriving some known eigenvalue error bounds using our identities. We now easily derive two known bounds, reviewed in section 2 , from the results obtained in section 3. First, since $\Sigma_{\min }(A) \leq \lambda_{\min }\left(A_{S}\right) \leq \lambda_{\max }\left(A_{S}\right) \leq \Sigma_{\max }(A)$, from (3.9) we obtain a priori bound (2.1),

$$
\begin{aligned}
|\lambda-\rho(y)| & =\left[\lambda_{\max }\left(A_{S}\right)-\lambda_{\min }\left(A_{S}\right)\right] \sin ^{2}(\angle\{x, y\}) \\
& \leq\left[\Sigma_{\max }(A)-\Sigma_{\min }(A)\right] \sin ^{2}(\angle\{x, y\}) .
\end{aligned}
$$

The inequality becomes an equality if $\Sigma_{\max }(A)$ and $\Sigma_{\min }(A)$ are eigenvalues of $A_{S}$. For details, see reference [7].

The second known result, mixed bound (2.2), follows from (3.10),

$$
\begin{aligned}
|\lambda-\rho(y)| & =\frac{\left\|P_{S} r(y)\right\|}{\|y\|} \tan (\angle\{x, y\}) \\
& \leq \frac{\|r(y)\|}{\|y\|} \tan (\angle\{x, y\}) .
\end{aligned}
$$

The inequality turns into an equality iff $P_{S} r(y)=r(y)$, i.e., iff $A y \in \operatorname{span}\{x, y\}$, which is equivalent to the subspace $\operatorname{span}\{x, y\}$ being $A$-invariant. More detailed information about the quality of this bound is determined by Remark 3.6 where vectors $x$ and $y$ are swapped to give us the following identity

$$
\|r(y)\| \cos (\angle\{r(y), x\})=\left\|P_{S} r(y)\right\| \cos \left(\angle\left\{P_{S} r(y), x\right\}\right) .
$$

REMARK 4.1. Our assumption that the operator $A$ is bounded is necessary in bound (2.1) and can be used in bound (2.2) to guarantee the existence of the vector Ay. Our results of section 3 formally speaking would hold for an unbounded operator $A$ and given vectors $x$ and $y$, as soon as the operator $A_{S}$ can be correctly defined by $A_{S}=\left.\left(P_{S} A\right)\right|_{S}$, where $S=\operatorname{span}\{x, y\}$. One could do even better than that, in fact, one only needs to be able to correctly define the values $\rho(x)$ and $\rho(y)$.

Rather than getting into technical details, we illustrate such a possibility using the following example from Davis and Kahan [2]. Let $\epsilon=1 / 2$ and $\mathcal{H}=l_{2}$. We take $y=\left(1, \epsilon, \epsilon^{2}, \ldots\right)^{T} \in l_{2}$ and $A=\operatorname{diag}\left(1, \epsilon^{-1}, \epsilon^{-2}, \ldots\right)$. We obtain $\rho(y)=1+\epsilon$ even though the sequence $A y=(1,1,1, \ldots)^{T}$ has an infinite norm in $l_{2}$.

Let us now consider $x=(1,0,0, \ldots)^{T} \in l_{2}$, the eigenvector corresponding to the lowest eigenvalue $\rho(x)=1$, and define $w=y-x=\left(0, \epsilon, \epsilon^{2}, \ldots\right)^{T} \in l_{2}$ with $\rho(w)=(1+\epsilon) / \epsilon=3$. Then $A_{S}$ can be constructed using its eigenvectors $u_{1}=w$ and $u_{2}=x$ and the corresponding eigenvalues $\mu=\rho(w)=3$ and $\nu=\rho(x)=1$ despite of the fact that the sequence $A w=(0,1,1, \ldots)^{T}$ does not exist in $\mathcal{H}=l_{2}$.

In the a priori bound, the eigenvalues of the operator $A_{S}$ are 1 and 3 , and our result (3.9) holds, while the bound (2.1) fails since $\Sigma_{\max }(A)=\infty$. In the mixed bound, we have $P_{S} r(y) \in l_{2}$ in our (3.10), but $\|r(y)\|=\infty$ in (2.2). For details, see the end of the next section where we return to this example.

We have now demonstrated how two known bounds, a priori bound (2.1) and mixed bound (2.2), can be derived in a simple and uniform manner from essentially two dimensional identities (3.9) and (3.10). The last well known bounds, we review in section 2, the a posteriori Temple bound (2.3) and its follow-ups, can also be derived in a similar way from their two dimensional prototype (3.3). However, the derivation is not so trivial, so we dedicate a separate section for it, coming next. 
5. New a posteriori bounds for eigenvalues. For convenience, let us remind the reader of the Temple bound (2.3),

$$
(\beta-\rho(y))(\rho(y)-\alpha) \leq \frac{\|r(y)\|^{2}}{\|y\|^{2}},
$$

where $\Sigma(A) \cap(\alpha, \beta)=\emptyset$, and its two dimensional analog (3.3), with $y$ substituted for $x$ for consistency of notation,

$$
\left[\lambda_{\max }\left(A_{S}\right)-\rho(y)\right]\left[\rho(y)-\lambda_{\min }\left(A_{S}\right)\right]=\frac{\left\|P_{S} r(y)\right\|^{2}}{\|y\|^{2}} .
$$

Our primary goal is to derive (2.3) from (3.3). We first cover an important particular case, where we can even improve (2.3).

LEMMA 5.1. Let $\alpha=\lambda_{\min }(A)$ be an isolated point of the spectrum of $A$ and $\beta>\alpha$ be the nearest larger point of the spectrum of $A$. Let $\mathcal{X}$ denote the complete eigenspace corresponding to the eigenvalue $\alpha$, and $P_{\mathcal{X}}$ be the orthogonal projector onto $\mathcal{X}$. For a vector $y \neq 0$, such that $\alpha<\rho(y)<\beta$, we define $x=P_{\mathcal{X}} y$ and $S=\operatorname{span}\{x, y\}$. Then

$$
(\beta-\rho(y))(\rho(y)-\alpha) \leq \frac{\left\|P_{S} r(y)\right\|^{2}}{\|y\|^{2}},
$$

where $P_{S}$ is the orthogonal projector on the subspace $S$.

Proof. The assumption $\rho(y)>\alpha$ implies $y \notin \mathcal{X}$ since $\alpha=\lambda_{\min }(A)$, so $x \neq y$. If $x=0$ then $y \perp \mathcal{X}$, but since $\mathcal{X}$ is the complete eigenspace corresponding to the eigenvalue $\alpha$, and $\beta>\alpha$ is the nearest larger point of the spectrum, $y \perp \mathcal{X}$ implies $\rho(y) \geq \beta$, which contradicts the lemma assumption $\rho(y)<\beta$. Thus $\operatorname{dim} S=2$ since $0 \neq x=P_{\mathcal{X}} y \neq y$, so our results of section 3 hold.

The vector $x$ is an eigenvector of $A_{S}$, and $\rho(x)=\lambda_{\min }(A)=\lambda_{\min }\left(A_{S}\right)$. Let $\mathcal{X}^{\perp}$ denote the orthogonal complement to $\mathcal{X}$. The vector $w=y-P_{\mathcal{X}} y \in S \cap \mathcal{X}^{\perp}$ is an eigenvector corresponding to the other eigenvalue of $A_{S}$, i.e., $\rho(w)=\lambda_{\max }\left(A_{S}\right)$. Since $\mathcal{X}$ is the complete eigenspace corresponding to the eigenvalue $\alpha=\lambda_{\min }(A)$, the fact that $w \in \mathcal{X}^{\perp}$ guarantees that $\beta \leq \rho(w)$. Therefore, collecting the bounds together, we get $\lambda_{\min }\left(A_{S}\right)=\alpha<\rho(y)<\beta \leq \rho(w)=\lambda_{\max }\left(A_{S}\right)$. Substituting $\beta \leq \lambda_{\max }\left(A_{S}\right)$ for $\lambda_{\max }\left(A_{S}\right)$ in identity (3.3) gives the desired bound.

The Temple bound (2.3), for the case of the smallest eigenvalue, follows from (5.1), using $\left\|P_{S} r(y)\right\| \leq\|r(y)\|$. In Remark 4.1 we have already discussed that having a bound based on $\left\|P_{S} r(y)\right\|$ rather than on $\|r(y)\|$ may provide great advantages. At the end of this section we extend the arguments of Remark 4.1 to investigate it.

Of course, Lemma 5.1 can be easily reformulated for the case of the opposite side of the spectrum of the operator $A$. Returning our attention to our main goal, we can construct simple examples showing that bound (5.1) does not hold if neither $\alpha$ nor $\beta$ corresponds to the extreme points of the spectrum of the operator $A$. The extension of bound (5.1) to the general case is described below. We start with a technical lemma.

Lemma 5.2. Let $\rho(y) \notin \Sigma(A)$ for a given vector $y \neq 0$. Let us denote by $U$ the invariant subspace of $A$ associated with all of the elements of the spectrum of $A$ larger than $\rho(y)$; and let us define

$$
V=U+\operatorname{span}\{y\}
$$

Then $\Sigma\left(A_{V}\right)=\rho(v) \cup \Sigma\left(A_{U}\right)$, and the smallest point $\Sigma_{\min }\left(A_{V}\right)<\rho(y)$ of the spectrum $\Sigma\left(A_{V}\right)$ is an isolated point - an eigenvalue of multiplicity one. 
Proof. Since $V=U+\operatorname{span}\{y\}$, we have $\operatorname{dim}\left(V \cap U^{\perp}\right)=1$. We define a nonzero vector $v=\left(I-P_{U}\right) y \in V \cap U^{\perp}$, and notice that $V=U \oplus \operatorname{span}\{v\}$ is an orthogonal sum. Since $U$ is an $A$-invariant subspace, it is also $A_{V}$-invariant. Then the decomposition $V=U \oplus \operatorname{span}\{v\}$ implies that $v$ is an eigenvector of the operator $A_{V}$, corresponding to the eigenvalue $\rho(v)$. Moreover, by the variational principle for the Rayleigh quotient, $\rho(v) \leq \rho(y)<\Sigma_{\min }\left(A_{U}\right)$, according to the definition of $U$. Thus, we deduce that $\Sigma\left(A_{V}\right)=\rho(v) \cup \Sigma\left(A_{U}\right)$, where $\rho(v)=\Sigma_{\min }\left(A_{V}\right)$ is an isolated point. The multiplicity of the eigenvalue $\rho(v)$ is one, because, again, $\operatorname{dim}\left(V \cap U^{\perp}\right)=1$.

We are now prepared to prove our main result in this section.

Theorem 5.3. Let $\alpha<\rho(y)<\beta$ where $\alpha$ and $\beta$ are the nearest points of the spectrum of $A$ to $\rho(y)$. Let $U$ be the invariant subspace of $A$ associated with all of the elements of the spectrum of $A$ larger than $\rho(y)$. We define $x=\left(I-P_{U}\right) y$ and $S=\operatorname{span}\{x, y\}$. Then

$$
(\beta-\rho(y))(\rho(y)-\alpha) \leq \frac{\left\|P_{S} r(y)\right\|^{2}}{\|y\|^{2}} .
$$

Proof. Applying Lemma 5.2 and noticing that $\Sigma_{\min }\left(A_{U}\right)=\beta$, we observe that the assumptions of Lemma 5.1 are satisfied if $A_{V}$ replaces $A$, where the scalar $\beta$ and the eigenvalue $\Sigma_{\min }\left(A_{V}\right)$ are the nearest points of the spectrum of the operator $A_{V}$ to $\rho\left(y, A_{V}\right)=\rho(y) \in\left(\Sigma_{\min }\left(A_{V}\right), \beta\right)$. Thus, by Lemma 5.1,

$$
(\beta-\rho(y))\left(\rho(y)-\Sigma_{\min }\left(A_{V}\right)\right) \leq \frac{\left\|P_{S} r\left(y, A_{V}\right)\right\|^{2}}{\|y\|^{2}}=\frac{\left\|P_{S} r(y)\right\|^{2}}{\|y\|^{2}} .
$$

Since $\Sigma_{\min }\left(A_{V}\right) \leq \alpha<\rho(y)$, we obtain (5.2).

REMARK 5.4. Substituting $-A$ for $A$ in Lemmas 5.1 and 5.2, and using similar arguments, we end up with the same subspace $S=\operatorname{span}\left\{P_{U} y, y\right\}=\operatorname{span}\left\{\left(I-P_{U}\right) y, y\right\}$ and obtain exactly the same bound.

Repeating the arguments of section 2, we obtain improved versions of the KatoTemple and other bounds, reviewed in section 2, from Theorem 5.3. Specifically, defining the subspace $S$ as in Theorem 5.3 and using the notation and assumptions of section 2, we get the improved Kato-Temple bound as follows,

$$
-\frac{1}{\rho(y)-a} \frac{\left\|P_{S} r(y)\right\|^{2}}{\|y\|^{2}} \leq \rho(y)-\lambda \leq \frac{1}{b-\rho(y)} \frac{\left\|P_{S} r(y)\right\|^{2}}{\|y\|^{2}}
$$

which, after introducing $\delta=\min _{\eta \in\{\Sigma(A) \backslash\{\lambda\}\}}|\eta-\rho(y)|$, implies

$$
|\lambda-\rho(y)| \leq \frac{1}{\delta} \frac{\left\|P_{S} r(y)\right\|^{2}}{\|y\|^{2}} .
$$

Theorem 5.3 also gives us the improved Krylov-Weinstein bound,

$$
|\lambda-\rho(y)| \leq \frac{\left\|P_{S} r(y)\right\|}{\|y\|} .
$$

Here, as in the original Krylov-Weinstein bound (2.6), only the existence of $\lambda \in \Sigma(A)$, which may or may not be an eigenvalue, is guaranteed.

REMARK 5.5. We note that $\left\|P_{S} r(y)\right\| \leq\left\|P_{V} r(y)\right\| \leq\|r(y)\|$, since $S \subseteq V \subset \mathcal{H}$. Thus, first, the classical a posteriori bounds now trivially follows from our bounds. 
Second, if the smallest quantity $\left\|P_{S} r(y)\right\|$ may not be readily accessible in a practical situation, it can be bounded above by the following much simpler expression, $\left\|P_{V} r(y)\right\|$, which can still be dramatically smaller compared to the standard value $\|r(y)\|$ used in the Temple bound (2.3). As an alternative for $V=U+\operatorname{span}\{y\}$ as defined in Lemma 5.2, we can take $V=U^{\perp}+\operatorname{span}\{y\} \supseteq S$.

As an illustration of possible improvements in $\left\|P_{S} r(y)\right\| \leq\left\|P_{V} r(y)\right\| \leq\|r(y)\|$, let us again, as in Remark 4.1, consider the example from Davis and Kahan [2] in $\mathcal{H}=l_{2}$. Let $\epsilon=1 / 2, y=\left(1, \epsilon, \epsilon^{2}, \ldots\right)^{T} \in l_{2}$, and $A=\operatorname{diag}\left(1, \epsilon^{-1}, \epsilon^{-2}, \ldots\right)$. We get $\rho(y)=1+\epsilon$ even though both the vector $A y=(1,1,1, \ldots)^{T}$ and the residual $r(y)=A y-\rho(y) y$ have infinite norms in $l_{2}$. Since $\|r(y)\|=\infty$, neither Temple bound (2.3), nor any other $\|r(y)\|$-based bound, can be used in this example, as Davis and Kahan correctly point out in [2, p. 42].

At the same time, let us choose the subspace $U$ as described in Theorem 5.3. Specifically, since $\epsilon=1 / 2$, we have $\rho(y)=1+\epsilon=1.5 \in(1,2)$, thus $U^{\perp}$ is simply the span of the first coordinate sequence $e_{1}=(1,0,0, \ldots)^{T}$, which is the eigenvector of $A$ corresponding to the smallest eigenvalue $\rho(x)=1$. This gives $x=\left(I-P_{U}\right) y=e_{1}$ and Theorem 5.3 turns into its particular case, Lemma 5.1. Since $S=\operatorname{span}\{x, y\}$, the sequence $x=e_{1}$ is an eigenvector of both operators $A$ and $A_{S}$ corresponding to the same smallest eigenvalue $\alpha=\lambda_{\min }(A)=\lambda_{\min }\left(A_{S}\right)=1$. Then the sequence $w=y-x=\left(0, \epsilon, \epsilon^{2}, \epsilon^{3}, \ldots\right)^{T}$ is the second eigenvector of $A_{S}$ corresponding to the eigenvalue $\rho(w)=\lambda_{\max }\left(A_{S}\right)=(1+\epsilon) / \epsilon=3$, while $\beta=1 / \epsilon=2$. Rather than explicitly calculating the sequence $P_{S} r(y)$ and its $l_{2}$ norm, we can take a short cut using Lemma 3.4,

$$
\begin{aligned}
\frac{\left\|P_{S} r(y)\right\|^{2}}{\|y\|^{2}} & =\left[\lambda_{\max }\left(A_{S}\right)-\rho(y)\right]\left[\rho(y)-\lambda_{\min }\left(A_{S}\right)\right] \\
& =\left[\frac{1+\epsilon}{\epsilon}-(1+\epsilon)\right][1+\epsilon-1] \\
& =1-\epsilon^{2}=\frac{3}{4}
\end{aligned}
$$

which gives the right-hand side of our bound (5.2). Its left-hand side is

$$
(\beta-\rho(y))(\rho(y)-\alpha)=\left(\frac{1}{\epsilon}-(1+\epsilon)\right)((1+\epsilon)-1)=1-\epsilon-\epsilon^{2}=\frac{1}{4} .
$$

We conclude that our bound $1 / 4 \leq 3 / 4$ holds, while the Temple bound fails, giving just the trivial statement $1 / 4 \leq \infty$.

By modifying the choice of the sequence $y$ in the example above, e.g., by choosing $y=\left(0,1, \epsilon, \epsilon^{2}, \ldots\right)^{T}$, one can construct similar situations, but where Theorem 5.3 is different from Lemma 5.1.

Let us finally comment that in this example our alternative choices for the subspace $V$, namely, $V=U+\operatorname{span}\{y\}$ as in Lemma 5.2 or $V=U^{\perp}+\operatorname{span}\{y\}$ as in Remark 5.5, give extremely different results. Evidently, the first choice leads to $V=H$ and gives no improvement compared to the broken Temple bound, while the second choice gives $V=S$ and works, as has already been shown above.

This example demonstrates an opportunity to easily treat unbounded operators. The unbounded case naturally appears for partial differential operators in the $L_{2}$ norm; see, e.g., [8] for a similar approach to analyze the Finite Element Method. 
6. Improving known error bounds for eigenvectors. The main topic of this work is RQ bounds. However, having the machinery of identities already constructed in section 3, we need only very little extra effort to revisit and improve some well known error bounds for eigenvectors, as a bonus. The reader might have noticed a couple of identities, (3.4) and (3.11), that have not yet been used. For convenience, we repeat them here, changing the notation in (3.4), for the case where $S=\operatorname{span}\{x, y\}$, the vector $x$ is an eigenvector of $A$ (and, thus, $A_{S}$ ) with the eigenvalue $\lambda=\rho(x)$, the scalar $\eta \neq \lambda$ denotes the other eigenvalue of $A_{S}$, and $\theta=\angle\{x, y\}$ :

$$
\sin (2 \theta)=\frac{2}{|\lambda-\eta|} \frac{\left\|P_{S} r(y)\right\|}{\|y\|}
$$

and, if $\theta<\pi / 2$,

$$
\tan (\theta)=\frac{1}{|\eta-\rho(y)|} \frac{\left\|P_{S} r(y)\right\|}{\|y\|} .
$$

In order to use identities (6.1) and (6.2), one needs to bound the scalar $\eta$. It is easily possible in the special case where $\lambda=\lambda_{\min }(A)$ or $\lambda=\lambda_{\max }(A)$. The latter choice is reduced to the former one by substituting $-A$ for $A$. We can use the arguments from the proof of Lemma 5.1 to handle the case $\lambda=\lambda_{\min }(A)$. Let the assumptions of Lemma 5.1 be satisfied, i.e., let $\alpha=\lambda_{\min }(A)$ be an isolated point of the spectrum of $A$ and $\beta>\alpha$ be the nearest larger point of the spectrum of $A$. Let $\mathcal{X}$ denote the complete eigenspace corresponding to the eigenvalue $\alpha$. For a given vector $y \neq 0$, such that $\alpha<\rho(y)<\beta$, we define $x=P_{\mathcal{X}} y$, where $P_{\mathcal{X}}$ is the orthogonal projector to the subspace $\mathcal{X}$; and we denote $S=\operatorname{span}\{x, y\}$. Then $\lambda=\rho(x)=\alpha$ and $\beta \leq \eta$, directly by the proof of Lemma 5.1. Substituting $\beta \leq \eta$ in (6.1) and (6.2), we obtain the following improvements of the corresponding bounds from [2, p. 11],

$$
\sin (2 \theta) \leq \frac{2}{\beta-\lambda} \frac{\left\|P_{S} r(y)\right\|}{\|y\|}
$$

and, if $\theta<\pi / 2$, from [2, p. 11] (see a single-vector version in [9, Corollary 11.7.1]),

$$
\tan (\theta) \leq \frac{1}{\beta-\rho(y)} \frac{\left\|P_{S} r(y)\right\|}{\|y\|} .
$$

The example from Davis and Kahan [2, p. 42], discussed above, is also applicable here, to observe that the use of the norm of the projected residual $\left\|P_{S} r(y)\right\|$ in our bounds can bring a dramatic improvement compared to the traditional term $\|r(y)\|$.

One final observation concerns the classical $\sin (\theta)$ bound, see, e.g., [2, p. 10] and, for a single-vector version that we use here, [1, Corollary 6.22] or [12, Theorem 3.9],

$$
\sin (\theta) \leq \frac{1}{\delta} \frac{\|r(y)\|}{\|y\|}
$$

where $\delta=\min _{\eta \in\{\Sigma(A) \backslash\{\lambda\}\}}|\eta-\rho(y)|$. Interestingly, an attempt of a naive improvement of (6.3), where $\left\|P_{S} r(y)\right\|$ simply substitutes for $\|r(y)\|$ without any other changes, fails as the following example demonstrates. Let $y=(1,1,1)^{T}$ and $A=\operatorname{diag}(1,0,-1)$. We get $A y=(1,0,-1)^{T}$ and $\rho(y)=0$, so $r(y)=A y$. Let $x=(0,1,0)^{T}$ with $\lambda=\rho(x)=0$. The residual $r(y)=(1,0,-1)^{T}$ is perpendicular both to $x$ and $y$, thus $P_{S} r(y)=0$, while $\delta=1$ and $\sin ^{2}(\theta)=2 / 3$. At the same time, $\|r(y)\|^{2} /\|y\|^{2}=2 / 3$, i.e., the original $\sin (\theta)$ bound (6.3) holds fine. The moral of this observation is that one cannot just blindly substitute $\left\|P_{S} r(y)\right\|$ for $\|r(y)\|$ and hope for improvement without breaking the original statement. 
Conclusion. We demonstrate the fundamental nature of our new RQ identities and concise inequalities, by using them to derive, in a unifying manner, and improve several known eigenvalue error bounds, including the famous Temple bound. The next natural step is to attempt to extend our single-vector RQ results to matrix RQ, thus hoping to simplify the derivation and to improve some classical results for the Rayleigh-Ritz method. We conjecture, by analogy with the approach of this paper, that the key to such a development is a careful sharp analysis of sensitivity of the Ritz values with respect to variations in the trial subspace in the Rayleigh-Ritz method.

Acknowledgments. We thank Ilse Ipsen, Dianne O'Leary, and anonymous referees for constructive comments that helped us to improve the presentation. We are grateful to SIMAX Associate Editor Michiel Hochstenbach for handling our paper.

\section{REFERENCES}

[1] F. Chatelin, Spectral Approximation of Linear Operators, Society for Industrial and Applied Mathematics (SIAM), Philadelphia, PA, 2011. doi:10.1137/1.9781611970678.

[2] C. Davis And W. M. Kahan, The rotation of eigenvectors by a perturbation. III, SIAM J. Numer. Anal., 7(1970), pp. 1-46. doi:10.1137/0707001.

[3] T. Kato, On the upper and lower bounds of eigenvalues, J.Phys. Soc. Japan, 4(1949), pp. 334339. doi:10.1143/JPSJ.4.334.

[4] A. V. KNYAZEv, Sharp a priori error estimates of the Rayleigh-Ritz method without assumptions of fixed sign or compactness, Math. Notes, 38(1986), pp. 998-1002. doi:10.1007/BF01157020.

[5] A. V. Knyazev, New estimates for Ritz vectors, Math. Comp., 66(1997), pp. 985-995. doi:10.1090/S0025-5718-97-00855-7.

[6] A. V. Knyazev, A. Jujunashvili and M. E. Argentati, Angles between infinite dimensional subspaces with applications to the Rayleigh-Ritz and alternating projectors methods, J. Funct. Anal., 259(2010), pp. 1323-1345. doi:10.1016/j.jfa.2010.05.018.

[7] A. V. Knyazev and M. E. Argentati, On proximity of Rayleigh quotients for different vectors and Ritz values generated by different trial subspaces, Linear Algebra Appl., 415(2006), pp. 82-95. doi:10.1016/j.laa.2005.06.032.

[8] A. V. Knyazev And M. E. ARgentati, Rayleigh-Ritz majorization error bounds with applications to FEM, SIAM J. Matrix Anal. Appl. 31(2009), 3, pp. 1521-1537. doi:10.1137/08072574X

[9] B. N. Parlett, The Symmetric Eigenvalue Problem, Society for Industrial and Applied Mathematics (SIAM), Philadelphia, PA, 1998. doi:10.1137/1.9781611971163.

[10] M. Reed And B. Simon, Analysis of Operators. Volume IV of Methods of Modern Mathematical Physics, Academic Press, 1978. ISBN 978-0125850049.

[11] A. RuHE, Computation of eigenvalues and eigenvectors, Lecture Notes in Math., 572(1977), pp. 130-184. doi:10.1007/BFb0116617.

[12] Y. SAAD, Numerical Methods for Large Eigenvalue Problems, Revised Edition, Society for Industrial and Applied Mathematics (SIAM), Philadelphia, PA, 2011. doi:10.1137/1.9781611970739.

[13] G. L.G. SleiJPen , J. VAn Den Eshof, On the use of harmonic Ritz pairs in approximating internal eigenpairs, Linear Algebra Appl. 358 (2003), pp. 115-137. doi:10.1016/S0024-3795(01)00480-3

[14] J. Sun, Eigenvalues of Rayleigh quotient matrices, Numer. Math., 59(1991), pp. 603-614. doi:10.1007/BF01385798.

[15] G. W. Stewart and J. G. Sun, Matrix Perturbation Theory, Computer Science and Scientific Computing, Academic Press Inc., Boston, MA, 1990, pp. xvi+365, ISBN 978-0126702309.

[16] G. Temple, The theory of Rayleigh's principle as applied to continuous systems, Proc. Roy. Soc. London. Ser. A., 119(1928), pp. 276-293. doi:10.1098/rspa.1928.0098.

[17] K. Yosida, Functional Analysis, Springer. Reprint of the 6th ed. Berlin, Heidelberg, New York 1980, 1995, XII, pp. 502. ISBN 978-3-540-58654-8.

[18] Y. Zhang, L. QIU, From subadditive inequalities of singular values to triangle inequalities of canonical angles, SIAM J. Matrix Anal. Appl. 31, 2009/10, 4, pp. 1606-1620. doi:10.1137/09074841X. 